\title{
Non-thermal histotripsy tumor ablation promotes abscopal immune responses that enhance cancer immunotherapy
}

To cite: Qu S, Worlikar T, Felsted AE, et al. Non-thermal histotripsy tumor ablation promotes abscopal immune responses that enhance cancer immunotherapy. Journal for ImmunoTherapy of Cancer 2020;8:e000200. doi:10.1136/ jitc-2019-000200

\section{- Prepublication history and} additional material for this paper are available online. To view these files, please visit the journal online (http://dx.doi.org/ 10.1136/jitc-2019-000200).

SQ, TW and AEF contributed equally.

ZX and CSC are Co-senior authors.

Accepted 23 November 2019

Check for updates

(C) Author(s) (or their employer(s)) 2020. Re-use permitted under CC BY-NC. No commercial re-use. See rights and permissions. Published by BMJ.

For numbered affiliations see end of article.

Correspondence to Dr Clifford Suhyun Cho; cliffcho@med.umich.edu

\author{
Shibin Qu, ${ }^{1,2}$ Tejaswi Worlikar, ${ }^{3}$ Amy E Felsted, ${ }^{1}$ Anutosh Ganguly, ${ }^{1,4}$ \\ Megan V Beems, ${ }^{1}$ Ryan Hubbard, ${ }^{3}$ Ashley L Pepple, ${ }^{1}$ Alicia A Kevelin, ${ }^{1}$ \\ Hannah Garavaglia, ${ }^{1}$ Joe Dib, ${ }^{1}$ Mariam Toma, ${ }^{1}$ Hai Huang, ${ }^{5}$ Allan Tsung, ${ }^{5}$ Zhen $\mathrm{Xu},{ }^{3}$ \\ Clifford Suhyun Cho ${ }^{1,4}$
}

\section{ABSTRACT}

Background Developing the ability to use tumor-directed therapies to trigger potentially therapeutic immune responses against cancer antigens remains a high priority for cancer immunotherapy. We hypothesized that histotripsy, a novel non-invasive, non-thermal ablation modality that uses ultrasound-generated acoustic cavitation to disrupt tissues, could engender adaptive immune responses to tumor antigens.

Methods Immunocompetent C57BL/6 mice inoculated with flank melanoma or hepatocellular carcinoma tumors were treated with histotripsy, thermal ablation, radiation therapy, or cytotoxic T lymphocyte-associated protein-4 (CTLA-4) blockade checkpoint inhibition. Lymphocyte responses were measured using flow cytometric and immunohistochemical analyses. The impact of histotripsy on abscopal immune responses was assessed in mice bearing bilateral tumors, or unilateral tumors with pulmonary tumors established via tail vein injection. Results Histotripsy ablation of subcutaneous murine melanoma tumors stimulated potent local intratumoral infiltration of innate and adaptive immune cell populations. The magnitude of this immunostimulation was stronger than that seen with tumor irradiation or thermal ablation. Histotripsy also promoted abscopal immune responses at untreated tumor sites and inhibited growth of pulmonary metastases. Histotripsy was capable of releasing tumor antigens with retained immunogenicity, and this immunostimulatory effect was associated with calreticulin translocation to the cellular membrane and local and systemic release of high mobility group box protein 1 . Histotripsy ablation potentiated the efficacy of checkpoint inhibition immunotherapy in murine models of melanoma and hepatocellular carcinoma.

Conclusions These preclinical observations suggest that non-invasive histotripsy ablation can be used to stimulate tumor-specific immune responses capable of magnifying the impact of checkpoint inhibition immunotherapy.

\section{BACKGROUND}

Recent advances in checkpoint inhibition immunotherapy have renewed investigative interest into the possibility that tumordirected therapies like thermal ablation and radiation could stimulate tumor-directed immune responses. By inciting inflammation or release of tumor antigens within the tumor microenvironment, ablation and radiation could theoretically potentiate the effects of checkpoint inhibition, even sensitizing previously resistant cancers to immunotherapy. ${ }^{1-8}$ Although immunostimulatory effects have been observed with thermal ablation and radiation, the magnitude of these effects has not yet proven capable of consistently augmenting the effect of immunotherapy. One potential immunostimulatory limitation of tumor-directed therapies may be their inability to induce sufficient tumorous release of immunogenic or inflammatory subcellular components, such as neoantigens or damageassociated molecular patterns (DAMPs) like high mobility group box protein 1 (HMGB1) that are capable of triggering strong tumordirected adaptive immune responses. ${ }^{9} 10$

Histotripsy is a novel modality of noninvasive tumor ablation that uses overlapping high-pressure ultrasound pulses to disrupt cellular architecture. At their point of convergence, focused ultrasound waves create precise regions of extreme pressure changes. Histotripsy uses microsecond-length ultrasound pulses to mechanically homogenize tissues through acoustic cavitation; by separating these pulses by milliseconds offtime or longer, heat generation is avoided. When applied to tumors, histotripsy reduces tumor tissue to a liquefied acellular homogenate that is gradually reabsorbed. ${ }^{11-18}$ By lysing target cells through a strictly mechanical mechanism that avoids the denaturing effects of heat or ionizing radiation, we hypothesized that histotripsy could promote inflammatory and immunostimulatory effects not possible with other modalities of tumordirected therapy like thermal ablation or radiation. In this report, we use a murine 
model of subcutaneous tumor ablation to demonstrate that histotripsy is uniquely capable of promoting local, regional, and systemic antitumor adaptive immune responses that can significantly augment the efficacy of checkpoint inhibition immunotherapy.

\section{METHODS}

\section{Mice and cell lines}

C57BL/6 mice (Mus musculus) aged 6-8 weeks old were purchased from Taconic (Hudson, New York) and housed and maintained in specific pathogen-free conditions. Each experiment involved the use of 4-23 mice per experimental group, and experimental group sizes are noted in the figure legends. As previously published, ${ }^{19-24}$ the B16GP33 cell line was established by transfecting B16F10, a poorly immunogenic melanoma cell line arising in C57BL/6 mice, with a plasmid encoding GP33, a class I major histocompatibility (MHC)-restricted lymphocytic choriomeningitis virus (LCMV) glycoprotein. Tumor inoculations were performed using B16GP33 melanoma and Hepa1-6 hepatocellular carcinoma (ATCC, Manassas, Virginia). B16GP33 cell lines were maintained by culturing with $200 \mu \mathrm{g} / \mathrm{mL}$ G418 in RPMI-1640 medium (Gibco, Life Technologies, Grand Island, New York) with $10 \%$ fetal bovine serum (HyClone, GE Healthcare Life Sciences), $2 \mathrm{mM} \mathrm{L-glutamine} \mathrm{(Gibco,} \mathrm{Life} \mathrm{Technologies),}$ $100 \mathrm{U} / \mathrm{mL}$ penicillin (Gibco, Life Technologies) and $100 \mu \mathrm{g} / \mathrm{mL}$ streptomycin (Gibco, Life Technologies), and Hepa 1-6cell lines were maintained by culturing in Dulbecco's modified Eagle's medium (DMEM) (Gibco, Life Technologies) with $10 \%$ fetal bovine serum.

\section{Tumor inoculation and monitoring}

Mice were inoculated by subcutaneous flank injection with $10^{6}$ B16GP33 or $4 \times 10^{6}$ Hepa1-6 cells suspended in phosphate buffered saline (PBS). Flank tumors were monitored and measured with electronic calipers every 3 days, and tumor volumes were calculated according to the following formula: volume=long dimension $\times$ short dimension $\times$ (short dimension/2). Pulmonary tumors were established by inoculating mice by tail vein injection with $2 \times 10^{5}$ B16GP33 cells suspended in PBS. Pulmonary metastases were quantified after euthanasia by counting the number of pigmented tumors identified along the surfaces of all lobes. Interobserver variability was mitigated by averaging the counts of three independent observers.

\section{In vivo tumor treatment}

Tumors were treated with histotripsy ablation, irradiation, radiofrequency ablation, or sham therapy. All procedures were performed following induction of isoflurane anesthesia. Histotripsy tumor ablation was performed by placing mice into a custom-built, eight-element, $1 \mathrm{MHz}$ focused ultrasound therapy transducer. A field-programmable gate array development board (DE0-Nano, Terasic Technology, Taiwan) controlled the therapy transducer to generate one to two cycles of histotripsy pulses. An L40-8/12 20 $\mathrm{MHz}$ imaging probe (Ultrasonix, Vancouver, Canada) was coaxially aligned with the therapy transducer to visualize the histotripsy bubble cloud and provide real-time targeting feedback. Using a motorized positioning system, the tumor was scanned to label treatment boundaries in three orthogonal dimensions. Boundary coordinates were used to generate a three-dimensional ellipsoid consisting of uniformly spaced treatment locations (spacing ranged from 0.4 to $0.7 \mathrm{~mm}$ in the axial, lateral, and elevational dimensions) to encompass the tumor while avoiding injury to surrounding tissues. At each location, 50 histotripsy pulses at $100 \mathrm{~Hz}$ pulse repetition frequency (PRF) were delivered to generate an estimated $-30 \mathrm{MPa}$ peak negative pressure at the focus, and all treatment locations were traversed by moving the therapy transducer using the motorized positioning system. Histotripsy duration ranged from 4 to $15 \mathrm{~min}$, depending on tumor volume and treatment location. Tumor irradiation was performed by positioning the mouse in radiation chambers with lead body shielding placed over the mouse such that only the tumor was exposed to 15 Gy unfractionated radiation. Radiofrequency ablation was performed by sterilizing the tumor site and passing an 18-gauge hollow needle into the tumor, through which a radiofrequency probe was passed for administration of thermal ablation at $90^{\circ} \mathrm{C}$ to encompass the tumor while avoiding injury to surrounding tissues. Sham therapy consisted of isoflurane anesthesia alone.

\section{In vitro CD8+ $\mathrm{T}$ cell stimulation}

Sham-ablated tumors were explanted and placed in $1.5 \mathrm{~mL}$ tubes and subjected to five cycles of rapid freezing in liquid nitrogen for $2 \mathrm{~min}$, followed by rapid thawing in a $60^{\circ} \mathrm{C}$ water bath for $2 \mathrm{~min}$. Non-ablated and in vivo histotripsy-ablated tumors were liquefied consistently and exposed to $10^{6} \mathrm{CD} 8+\mathrm{T}$ cells harvested from the spleen of C57BL/ 6 mice 8 days after LCMV infection (at which time $5 \%-10 \%$ of circulating CD8+ $\mathrm{T}$ cells are specific for GP33) in the presence of $10 \mathrm{U}$ interleukin (IL)-2 and brefeldin $\mathrm{A}$ at $37^{\circ} \mathrm{C}$ for 5 hours. CD8+ T cells were also exposed to media alone as negative controls and to $0.01 \mu \mathrm{g} / \mathrm{mL}$ GP33 peptide alone as positive controls.

\section{Checkpoint inhibition immunotherapy}

Checkpoint inhibition immunotherapy was performed by treating tumor-bearing mice with intraperitoneal injections of $200 \mu \mathrm{g}$ anti-cytotoxic $\mathrm{T}$ lymphocyte-associated protein-4 (CTLA-4) mAb (Bio X Cell, West Lebanon, New Hampshire) on days 6, 9 and 12 after B16GP33 tumor inoculation or days 3, 6, 9 and 12 after Hepa1-6 tumor inoculation.

\section{Flow cytometry}

Tumors, tumor-draining lymph nodes (TDLN), nontumor-draining lymph nodes (NTDLN), and spleens were harvested and processed into single-cell suspensions on sterile fine mesh screens. Tumor infiltrating lymphocytes (TIL) were isolated from dissociated tumors 
using Histopaque (Sigma Aldrich, St Louis, Missouri) centrifugation. Lymphocytes from each sample were stained with allophycocyanin (APC)-labeled MHC class I $\left(\mathrm{D}^{\mathrm{d}}\right)$ tetramers loaded with MHC class I-restricted GP33 peptide (National Institutes of Health Tetramer Core Facility, Atlanta, Georgia), Pacific Blue-labeled anti-CD8 mAb, BV510-labeled anti-CD4 mAb, PerCP/Cy5.5-labeled anti-CD19 mAb, PE/Cy7-labeled anti-CD11c mAb, AF488labeled anti-Ly6G mAb, BV650-labeled anti-NK1.1 mAb, and PE-labeled anti-CD25 mAb. For intracellular staining, cells were fixed, permeabilized, and stained with $\mathrm{PE}$ CF594-labeled anti-FoxP3 mAb or PE-labeled anti-IFN $\gamma$ mAb. All antibodies were purchased from BioLegend (San Diego, California), except for anti-CD8 (BD Biosciences, San Diego, California) and anti-Ly6G and antiFoxP3 (eBioscience, San Diego, California). Fluorescence data were acquired on a BD LSRFortessa Flow Cytometer (BD Biosciences) and analyzed using FlowJo software (Tree Star, Ashland, Oregon). All flow cytometry data are shown following gating on lymphocytes gate.

\section{Immunohistochemistry}

Tumors were washed with PBS and preserved in $10 \%$ formalin for 24-48 hours and in 70\% ethanol until paraffin embedding. Sections of $4.5 \mu \mathrm{m}$ thickness were deparaffinized in xylene for $6 \mathrm{~min}$, then washed in $50 \%$ xylene in ethanol and $100 \%$ ethanol, and rehydrated by sequential transfer to $95 \%, 70 \%$, and $50 \%$ ethanol, and ultimately to distilled water for $3 \mathrm{~min}$ each before staining. Samples were washed in PBS, blocked in $1 \%$ bovine serum albumin for $30 \mathrm{~min}$, and incubated with rabbit polyclonal antiCD8a antibody to CD8a antibody (synaptic system) at $4^{\circ} \mathrm{C}$ overnight. Samples were then washed and blocked in goat serum, incubated with biotin-conjugated goat antirabbit IgG for $45 \mathrm{~min}$, washed for $30 \mathrm{~min}$ in PBS, then incubated with horseradish peroxidase (HRP)-conjugated avidin. Samples were developed using the CN/DAB Substrate Kit (Thermo Fisher, Waltham, Massachusetts) for 5 min as per vendor instructions, then counterstained with hematoxylin for $10 \mathrm{~s}$, and washed and mounted. Samples were visualized using a $10 \times$ or $20 \times$ objective on a Nikon Eclipse E400 microscope, and images were captured using a DXM1200F camera using the software provided by the vendor (Nikon, Melville, New York). At least 10 images from each tumor sample were obtained for each magnification. Cells were counted using ImageJ software after appropriate background subtraction.

\section{Immunofluorescence}

Formalin-fixed paraffin-embedded tumor sections were deparaffinized and rehydrated as above. Heat-mediated antigen retrieval was performed in Tris buffer containing $1 \%$ Triton X (for CD8 staining) or citrate buffer (for calreticulin (CRT) staining) in a boiling water bath for $20 \mathrm{~min}$. Sections were blocked in $5 \%$ bovine serum albumin (BSA) in PBS for $30 \mathrm{~min}$, then primary antibody staining was performed with a primary rabbit IgG antibody against CD8, or rabbit IgG against CRT directly conjugated to
Alexa 647 and rabbit IgG against ERp72 directly conjugated to Alexa 555 (Abcam, Cambridge, England) overnight at $4^{\circ} \mathrm{C}$ overnight. Secondary antibody staining (for CD8 staining) was performed with Alexa 488-labelled goat antirabbit IgG for 1 hour. Sections were washed and mounted using Prolong Gold Antifade Mountant with 4'6-diamidino-2-phenylindone (DAPI) (Thermo Fisher), and a minimum of five overlapping optical fields from two independent samples were captured. Images were acquired using a Keyence BZ-800 microscope (Keyence, Osaka, Japan) under $10 \times$ objective with a $1 \times$ digital zoom, or under $40 \times$ objective with a $1 \times$ digital zoom. Images were imported to Image J and the threshold was adjusted using the color threshold tool.

\section{HMGB1 assays}

Serum levels of HMGB1 were measured by ELISA (Tecan, Männedorf, Switzerland), according to the manufacturer's instructions. Intratumoral levels of HMGB1 were measured by immunofluorescence using tumor samples snap-frozen in liquid nitrogen. Tumors were prepared on round cover glass and fixed in $4 \%$ paraformaldehyde (PFA) at room temperature for $15 \mathrm{~min}$ after PBS washing. Slides were permeabilized in $0.3 \%$ Triton X-100 for 10 min followed by blocking solution (5\% BSA in PBS) for $30 \mathrm{~min}$ at room temperature. Samples were stained in antibody reaction buffer ( $1 \%$ BSA and $0.3 \%$ Triton X-100 in PBS, $\mathrm{pH}$ 7.4) with a primary antibody for HMGB1 overnight at $4^{\circ} \mathrm{C}$, followed by secondary antibodies for 1 hour at room temperature. F-actin was stained with rhodamine phalloidin (Thermo Fisher). Hoechst 33342 (Thermo Fisher) was used for nuclear staining. All slides were scanned under the same conditions for magnification, exposure time, lamp intensity, and camera gain. Confocal images were acquired using an Olympus FluoView 1000 microscope (Olympus, Tokyo, Japan). All images were quantitated as previously described with HMGB1 staining intensity normalized to nuclei using MetaMorph software (Molecular Devices, Downingtown, Pennsylvania). ${ }^{25}$

\section{Statistical analysis}

Experimental data were analyzed using SPSS V.23 statistical software. Two groups of independent samples were compared using the two-tailed Student's t-test. Analysis of variance was applied to assess the statistical significance in tumor growth kinetics. Differences in survival between groups were assessed using the log-rank test. Significance was defined as $\mathrm{p}<0.05$, and error bars represent SEM.

\section{RESULTS}

Histotripsy promotes local intratumoral innate and adaptive immune responses

We examined the effect of histotripsy on intratumoral lymphocyte infiltration. Ten days after inoculation with subcutaneous flank injections of poorly immunogenic B16GP33 melanoma (a B16F10 melanoma cell line engineered to stably express very low levels of the LCMV peptide GP33 ${ }^{19-24}$ ), 
A

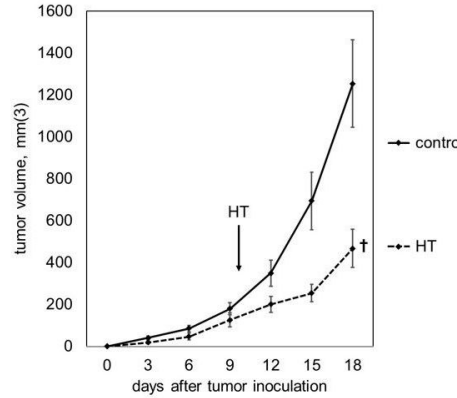

B

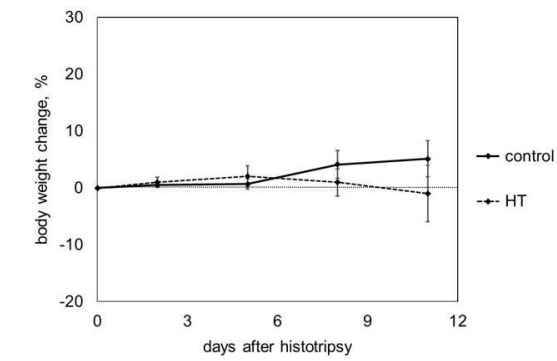

C

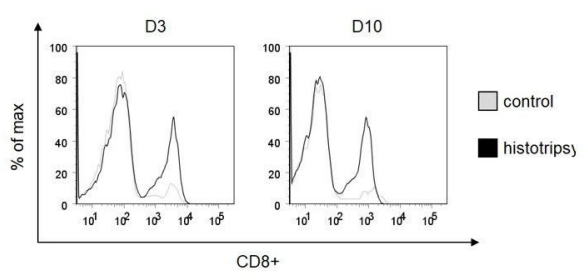

D

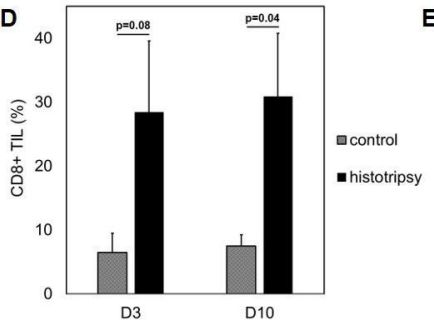

$y 6 G+$ TIL

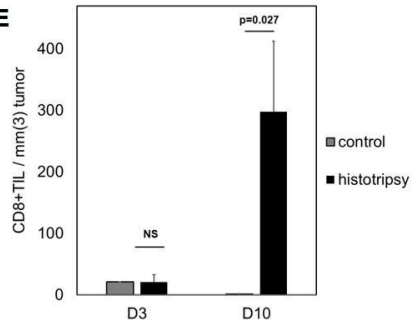

CD19+ TIL
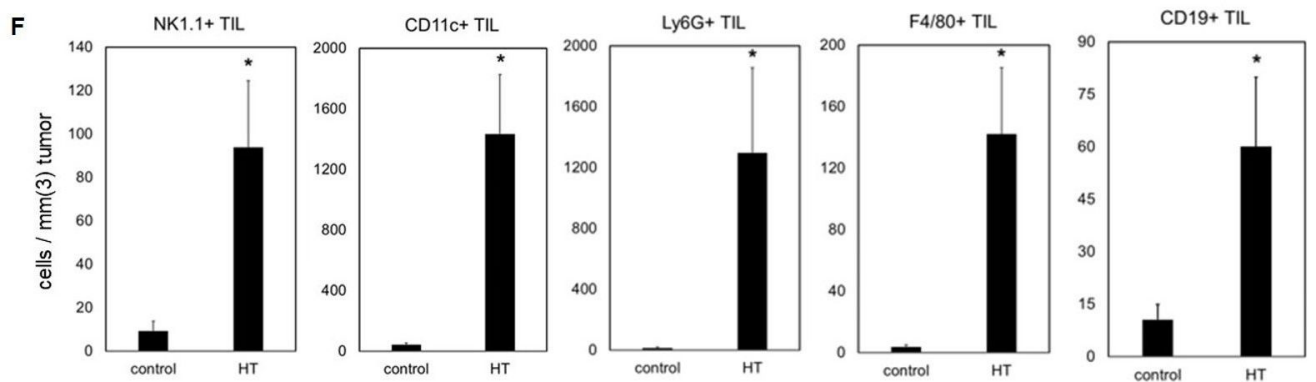

G
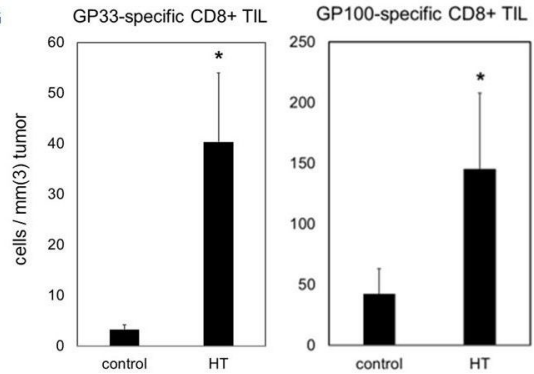

$\mathrm{H}$

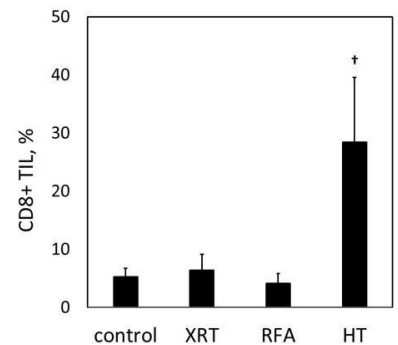

Figure 1 Histotripsy inhibition of tumor growth is well tolerated and promotes potent intratumoral CD8+ Tcell infiltration. C57BL/6 mice were inoculated with B16GP33 subcutaneous tumors, then treated with sham (control) or histotripsy ablation (HT) on day 10. Subtotal histotripsy ablation resulted in durable suppression of tumor growth (A). No significant differences in body weight were observed following histotripsy ablation (B). Fluorescence-activated cell sorting (FACS) analysis of tumorinfiltrating lymphocytes (TIL) on day 3 (D3) and day 10 (D10) after treatment (gated on viable lymphocytes) demonstrated higher percentages of intratumoral CD8+ Tcell infiltration after histotripsy by day 10 (C,D). Stimulation of CD8+ TIL remained significant on day 10 when cell numbers were normalized to tumor volume (E). FACS analysis of TIL on day 10 after treatment also identified marked increases in intratumoral populations of NK1.1+ natural killer cells, CD11c+ dendritic cells, Ly6G+ neutrophils, F4/80+ macrophages, and CD19+ B cells after histotripsy (F). Staining with major histocompatibility complex (MHC) tetramers loaded with the minor melanoma antigen GP33 and the major melanoma antigen GP100 identified significant increases in intratumoral populations of melanoma antigen-specific CD8+T cells (G). In contrast to histotripsy ablation (HT), tumor irradiation (XRT) and radiofrequency ablation (RFA) did not potentiate intratumoral CD8+ Tcell infiltration $(H)(n=4-8$ per group; $\nmid p<0.05$ vs all other groups by analysis of variance; ${ }^{*} \mathrm{p}<0.05$ vs control by t-test).

C57BL/6 mice were treated with sham or histotripsy ablation. Histotripsy ablation significantly inhibited tumor growth without producing significant alterations in body weight (figure $1 \mathrm{~A}, \mathrm{~B})$. On days 3 and 10 after sham or histotripsy ablation, mice were euthanized and tumors were harvested for flow cytometric analysis. As shown in figure 1C-F a significant upregulation of intratumoral natural killer (NK) cells, dendritic cells (DCs), neutrophils, B cells, CD4+ T cells and CD8+ T cells was observed following histotripsy ablation. In addition, histotripsy was also associated with significant intratumoral infiltration of melanoma antigen-specific CD8+ T cells (figure 1G).

Histotripsy mediates stronger intratumoral CD8+ T cell infiltration than other modalities of tumor-directed therapy We compared the magnitude of histotripsy-induced intratumoral $\mathrm{T}$ cell infiltration with that of radiation and thermal ablation. Ten days after inoculation with subcutaneous 

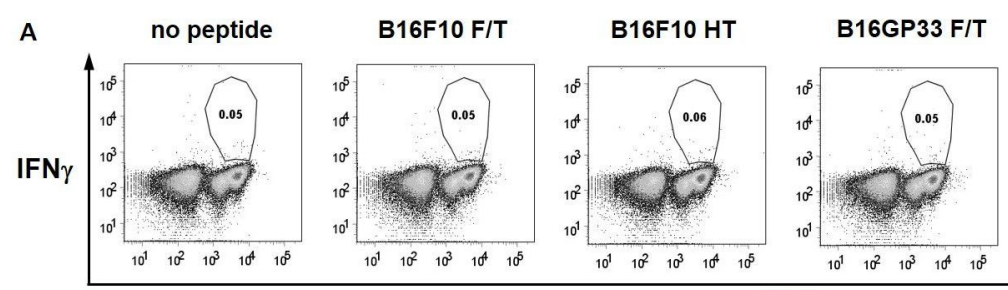

B16GP33 HT

GP33 peptide
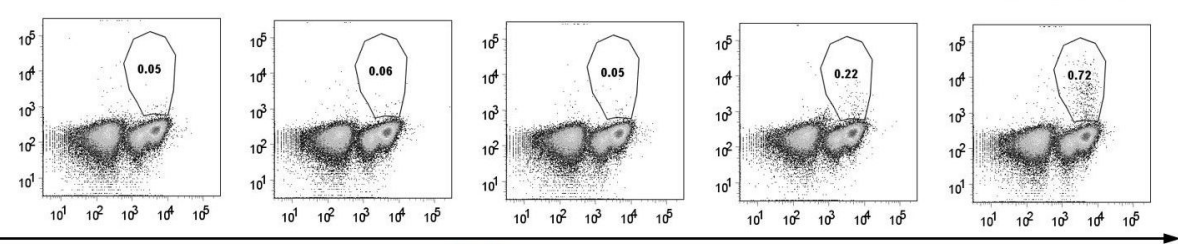

CD8

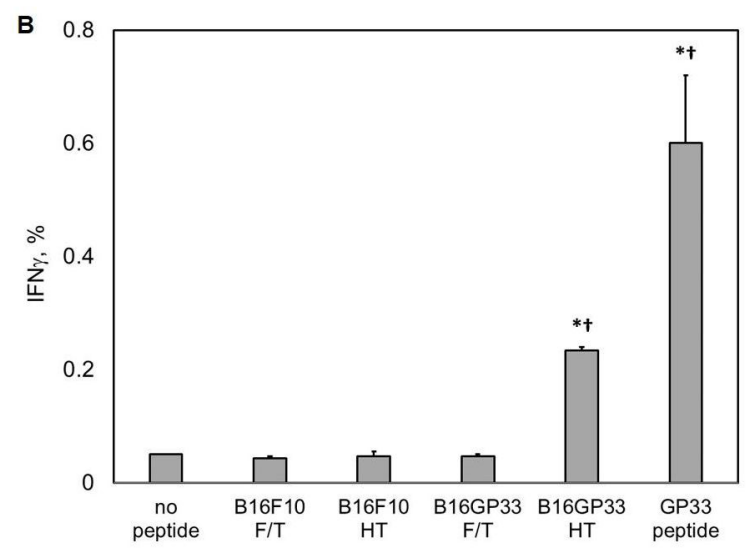

Figure 2 Non-thermal histotripsy is capable of releasing immunogenic tumor neoantigens. C57BL/6 mice were inoculated with B16F10 or B16GP33 subcutaneous tumors, then treated with sham or histotripsy ablation (HT) on day 10. Tumors were excised and sham-treated tumors were liquefied using three freeze-thaw (F/T) cycles consisting of 2 min in liquid nitrogen and $2 \mathrm{~min}$ in a $60^{\circ} \mathrm{C}$ water bath. Liquefied tumors were exposed to CD8+ T cells isolated from the spleens of C57BL/6 mice 8 days after LCMV infection (at which time small populations of GP33-specific effector CD8+ T cells exist) in the presence of interleukin-2 and brefeldin A. Negative controls consisted of CD8+ T cells alone (no peptide) and positive controls consisted of CD8+ T cells exposed to $0.01 \mu \mathrm{g} / \mathrm{mL}$ GP33 peptide (GP33 peptide). After 5-hour incubation, individual (A) and group (B) fluorescence-activated cell sorting (FACS) analyses of intracellular IFN $\gamma$ expression (gated on viable lymphocytes) demonstrated that exposure to histotripsy-treated B16GP33 tumors was the only experimental condition capable of stimulating GP33-specific CD8+ T cells in vitro. The magnitude of this stimulation was approximately half of that seen with direct exposure to GP33 peptide ( $n=4$ per group, ${ }^{*} p<0.05$ vs negative control by $t$-test; $t p<0.05$ vs all other experimental groups by analysis of variance). IFN $\gamma$, interferon gamma; LCMV, lymphocytic choriomeningitis virus.

B16GP33 melanoma tumors, C57BL/6 mice were treated with no therapy or single unfractionated treatments of 15 Gy external beam radiation, radiofrequency ablation, or histotripsy. As shown in figure $1 \mathrm{H}$, flow cytometric analysis of tumor-infiltrating lymphocytes isolated 10 days after treatment demonstrated that histotripsy alone resulted in significant increases in intratumoral CD8+ Tcell populations.

\section{Non-thermal histotripsy is capable of releasing immunogenic tumor neoantigens}

To explore the ability of histotripsy to release intact tumor antigens capable of stimulating CD8+ Tcell responses, subcutaneous $\mathrm{B} 16 \mathrm{~F} 10$ and B16GP33 melanoma tumors were explanted immediately after sham or histotripsy ablation performed 10 days after inoculation. Sham-ablated tumors were subjected to five cycles of rapid freezing and heating (consisting of exposure to liquid nitrogen followed by exposure to a $60^{\circ} \mathrm{C}$ water bath). CD8+ T cells isolated from splenocytes of C57BL/6 mice 8 days after LCMV infection (at which time a small percentage of CD8+ T cell populations are specific for the LCMV peptide GP33) were exposed to media (negative control), B16F10 or B16GP33 tumors treated with freezeheat, B16F10 or B16GP33 tumors treated with histotripsy, or $0.01 \mu \mathrm{g} / \mathrm{mL}$ GP33 peptide (positive control) in the presence of IL-2 and brefeldin. As shown in figure 2, activation of $\mathrm{CD} 8+\mathrm{T}$ cells (as indicated by intracellular interferon gamma (IFN $\gamma$ ) expression) was only observed after exposure to B16GP33 melanoma tumors treated with histotripsy. The magnitude of this CD8+ Tcell stimulation approached the level of stimulation seen with direct exposure to GP33 peptide (positive control), indicating that non-thermal disruption of tumors by histotripsy was capable of releasing preserved tumor antigens capable of stimulating CD8+ Tcell responses. The absence of CD8+ Tcell stimulation by B16F10 tumors (which do not express GP33) treated with histotripsy confirmed the antigen-specific nature of this response.

\section{Histotripsy is associated with regional and systemic tumor- specific CD8+ $T$ cell responses}

We examined the effect of histotripsy ablation on other lymphoid compartments beyond the treated tumor. Ten days after sham or histotripsy ablation of B16GP33 melanoma tumors, ipsilateral inguinal lymph nodes were harvested as TDLN, and contralateral axillary lymph nodes were harvested as NTDLN. As shown in online supplementary figure 1 , flow cytometric analysis identified slightly higher numbers of CD8+ T cells specific for the 
A

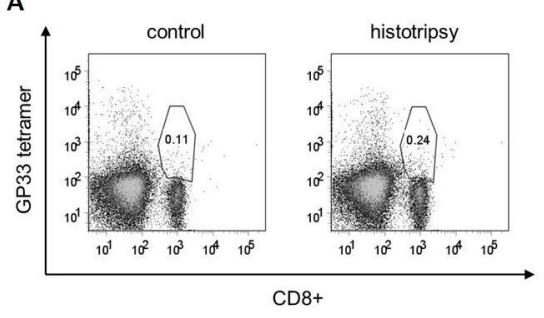

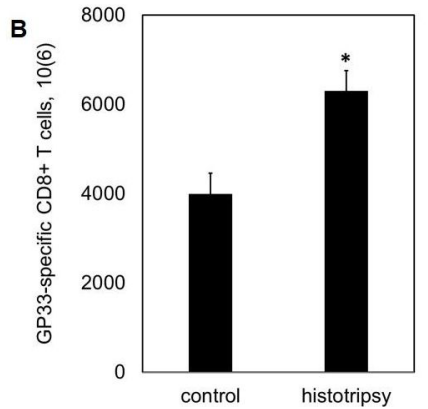

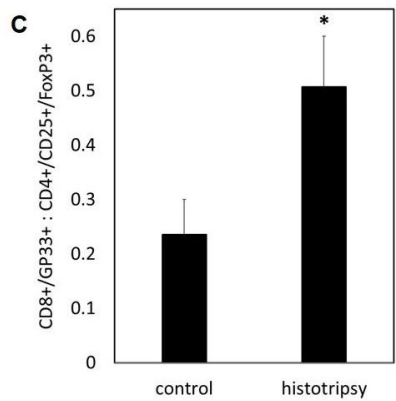

Figure 3 Histotripsy promotes regional and systemic tumor-specific CD8+ Tcell responses. C57BL/6 mice were inoculated with B16GP33 subcutaneous tumors expressing very low levels of GP33 peptide, then treated with no therapy (control), tumor irradiation (XRT), radiofrequency ablation (RFA), or histotripsy ablation on day 10. 10 days later, fluorescence-activated cell sorting (FACS) analyses of splenocytes (gated on viable lymphocytes) demonstrated higher numbers of circulating CD8+ T cells with T cell receptor (TCR) specificity for the minor melanoma antigen GP33 after histotripsy but not after XRT or RFA (A,B). Histotripsy was also associated with a higher ratio of circulating melanoma-specific CD8+ T cells to regulatory CD4+ $T$ cells $(C)$ ( $n=4$ per group, ${ }^{*} p<0.05$ vs control by t-test).

weakly immunogenic tumor antigen GP33 within TDLN but not NTDLN after histotripsy, but this did not reach statistical significance. However, analysis of splenocytes did identify significantly higher numbers of circulating GP33-specific CD8+ T cells after histotripsy as compared with controls, radiation, and radiofrequency ablation (figure 3A,B). Analysis of CD4+/CD25+/FoxP3+ cells demonstrated a favorable skewing of tumor-specific CD8+ $\mathrm{T}$ cell:regulatory $\mathrm{T}$ cell ratios after histotripsy (figure 3C).

\section{Histotripsy is associated with the induction of abscopal intratumoral CD8+ T cell responses}

Because of the unique ability of histotripsy to trigger significant local and systemic tumor-directed CD8+ Tcell responses, we evaluated the effect of histotripsy on distant tumor sites. Ten days after inoculation with bilateral subcutaneous flank B16GP33 melanoma tumors, C57BL/6 mice were treated with no therapy or with single unfractionated treatments of $15 \mathrm{~Gy}$ external beam radiation, radiofrequency ablation, or histotripsy ablation of unilateral tumors. Ten days after treatment, contralateral (non-treated) tumors were harvested for lymphocyte assays. As shown in figure 4A,B, flow cytometric analysis identified significantly higher levels of abscopal CD8+ Tcell infiltration into distant tumor sites after contralateral histotripsy ablation, but not after radiation therapy or radiofrequency ablation. Comparison between bilateral tumors using immunohistochemistry confirmed minimal infiltration of CD8+ $\mathrm{T}$ cells in control mice (figure 4C). Whereas tumors treated with histotripsy ablation exhibited brisk CD8+ Tcell infiltration along the periphery of ablation zones, abscopal CD8+ Tcell infiltration in contralateral tumors after histotripsy ablation was qualitatively different, exhibiting a more diffuse and homogeneous pattern (figure 4D,E). CD8+ T cell infiltration on days 3,5 and 7 after histotripsy was analyzed by immunohistochemistry. In control tumors, CD8+ T cell numbers were low and decreased over time; in histotripsy-ablated tumors, CD8+ Tcell numbers increased dramatically from day 3 to day 5 , and remained high on day 7 . The abscopal effect in contralateral tumors after histotripsy was characterized by a more gradual increase in CD8+ Tcell numbers (figure 4F). Of note, the ability of unilateral histotripsy ablation to promote distant CD8+ TIL was associated with a small but significant inhibition of contralateral tumor growth. In contrast, no abscopal inhibition of contralateral tumor growth was observed with radiation or radiofrequency ablation (figure 4G-I). Furthermore, despite the presence of a contralateral untreated tumor, survival studies indicated that the abscopal impact of unilateral histotripsy ablation was associated with a statistically significant survival advantage to mice bearing bilateral B16GP33 melanoma tumors (figure 4J).

\section{Histotripsy inhibits the development of distant metastases}

Given the evidence of abscopal immune stimulation, we tested the ability of local histotripsy ablation to inhibit growth of distant tumors. C57BL/6 mice were inoculated with unilateral subcutaneous injections of B16GP33 melanoma cells on day 0 and with intravenous injections of B16GP33 melanoma cells on day 3 to establish flank and pulmonary melanoma tumors, respectively. On day 10 , mice were treated with or without histotripsy ablation of flank tumors; on day 20, mice were euthanized for visual quantitation of pulmonary metastases. As shown in figure 5A,B, significantly fewer pulmonary metastases were observed in mice treated with histotripsy ablation of flank tumors. Immunohistochemical analysis demonstrated smaller tumor size and marked CD8+ Tcell infiltration among pulmonary metastases after flank tumor histotripsy ablation (figure 5C).

\section{Histotripsy is associated with the induction of systemic inflammatory changes and local release of HMGB1}

We hypothesized that the immunostimulatory effects of histotripsy could be associated with an ability to promote the release of intratumoral HMGB1. As illustrated in figure $1 \mathrm{~F}$, histotripsy tumor ablation was followed by an intense inflammatory infiltrate of NK cells, DCs, neutrophils and macrophages. As shown in figure $6 \mathrm{~A}-\mathrm{C}$, 

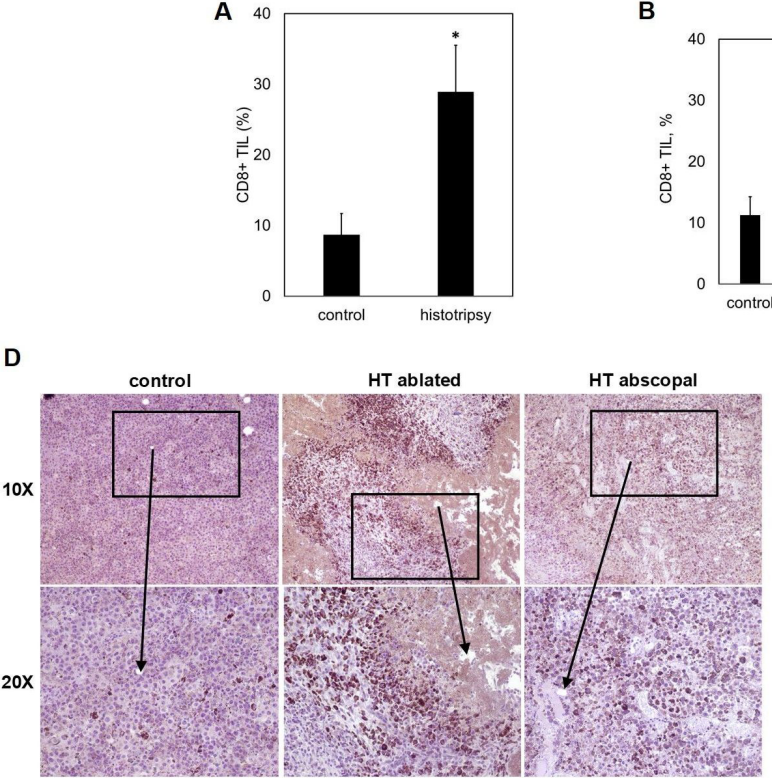

B

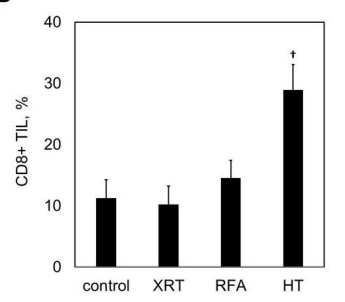

C
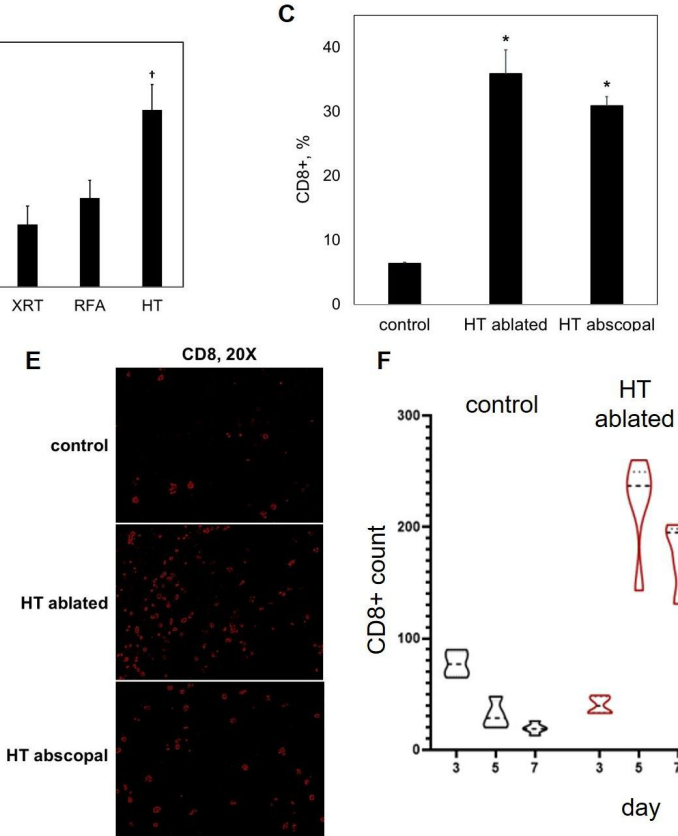

$\mathrm{F}$

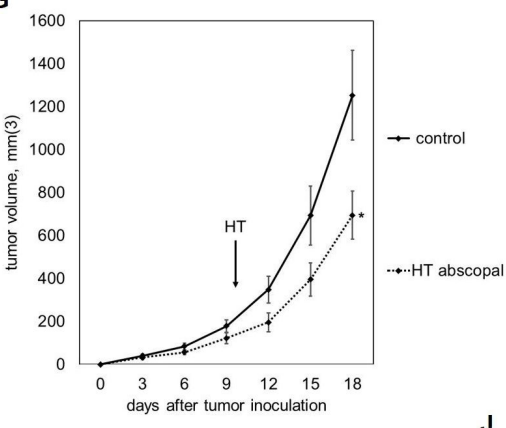

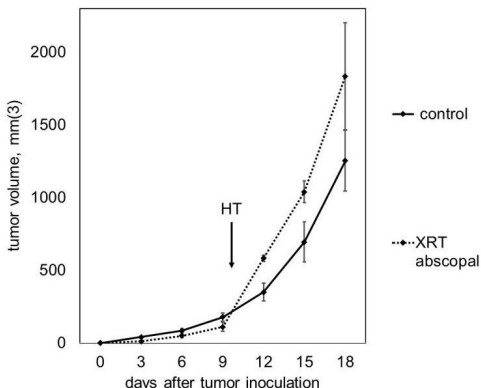
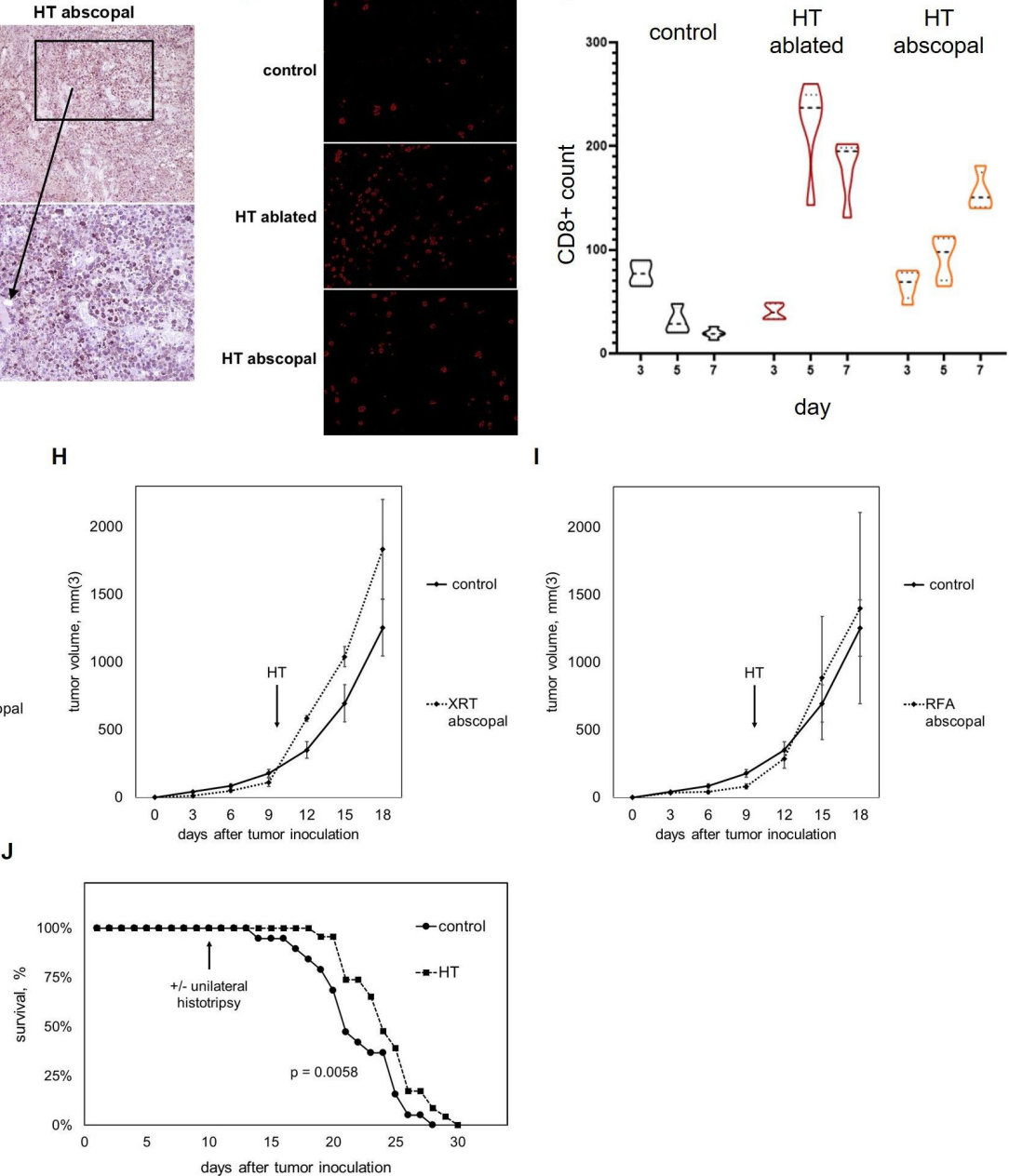
days after tumor inoculation

Figure 4 Histotripsy promotes abscopal CD8+ Tcell responses. 10 days after bilateral B16GP33 melanoma tumor inoculation, C57BL/6 mice were treated with unilateral sham (control) or histotripsy tumor ablation. Fluorescence-activated cell sorting (FACS) analysis of tumor infiltrating lymphocyte (TIL) populations 10 days after treatment (gated on viable lymphocytes) identified stronger intratumoral CD8+ T cell infiltration in contralateral, untreated tumors after histotripsy (A). In contrast to histotripsy ablation (HT), unilateral tumor irradiation (XRT) and radiofrequency ablation (RFA) did not promote abscopal infiltration of CD8+ TIL (B). FACS analysis of histotripsy-ablated (HT ablated) and contralateral non-ablated tumors (HT abscopal) identified comparable levels of intratumoral CD8+ T cell infiltration (C). Immunohistochemistry (D) and immunofluorescence staining (E) of CD8 confirmed similar levels of CD8+ T cell infiltration between histotripsy-ablated (HT ablated) and contralateral non-ablated tumors (HT abscopal), but with different patterns of infiltration. Whereas CD8+ T cells were largely localized to peripheral areas that surrounded ablation zones in histotripsy-ablated tumors, CD8+ Tcell infiltration was more diffuse and homogeneous in contralateral non-ablated tumors (D). Quantitation of CD8+ T cells using immunohistochemistry (defined as the average number of CD8+ T cells per $500 \mu \mathrm{m}^{2}$ area for a minimum of five non-overlapping fields from two independent samples) on days 3, 5 and 7 after sham or histotripsy ablation showed small and diminishing CD8+ Tcell numbers over time in control tumors, rapidly increasing CD8+ Tcell numbers in histotripsy-ablated tumors, and gradually increasing CD8+ Tcell numbers in contralateral non-ablated tumors (F). Comparison of tumor growth between treated and contralateral non-treated tumors showed that histotripsy was associated with a modest but significant abscopal inhibition of contralateral tumor growth $(\mathrm{G})$. In contrast, no abscopal effects were observed after unilateral irradiation $(\mathrm{H})$ or radiofrequency ablation (I). This abscopal inhibition of contralateral tumor growth by histotripsy was associated with significant prolongation of survival $(J)$ ( $n=4$ per group and 23 per group in survival experiments, ${ }^{*} p<0.05$ vs control by t-test; $\dagger p<0.05$ vs all other groups by analysis of variance). 
A

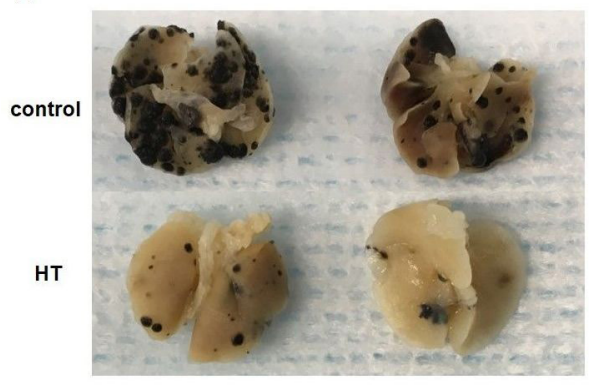

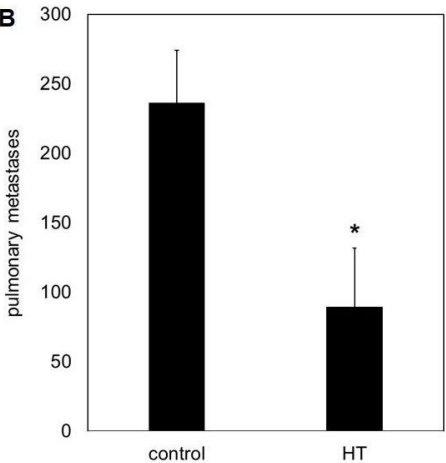

C
20X

control

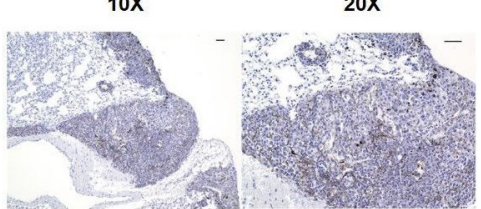

HT

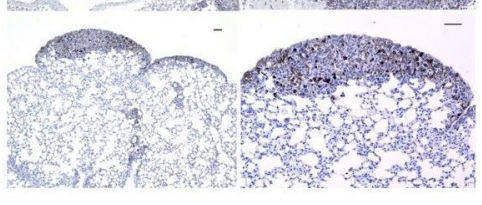

Figure 5 Histotripsy inhibits the development of distant pulmonary metastases. C57BL/6 mice were inoculated with flank and intravenous inoculations of B16GP33 melanoma on days 0 and 3, respectively. Sham (control) or histotripsy (HT) of flank tumors was performed on day 10 , and pulmonary metastases were quantified by visual inspection on day 20 . Representative specimens $(A)$ and group data $(B)$ demonstrated significantly fewer pulmonary metastases in mice treated with flank tumor histotripsy ablation. Immunohistochemical analysis demonstrated smaller tumor size and more concentrated CD8+ Tcell infiltration among pulmonary metastases in mice treated with histotripsy $(C)(n=4$ per group, * $p<0.05$ vs control by t-test; black bars indicate $50 \mu \mathrm{m})$.

flow cytometric analysis of splenocytes on day 10 after histotripsy demonstrated a significant increase in circulating NK cells and a trend toward higher percentages of circulating neutrophils. One day after histotripsy treatment, immunofluorescence assays identified apoptotic changes within treated tumors, as evidenced by nuclear condensation; this was associated with marked translocation of CRT from the endoplasmic reticulum to the plasma membrane as compared with untreated controls (figure 6D). On days 1 and 7 after histotripsy treatment, immunofluorescence assays demonstrated a significant extranuclear translocation of HMGB1 within
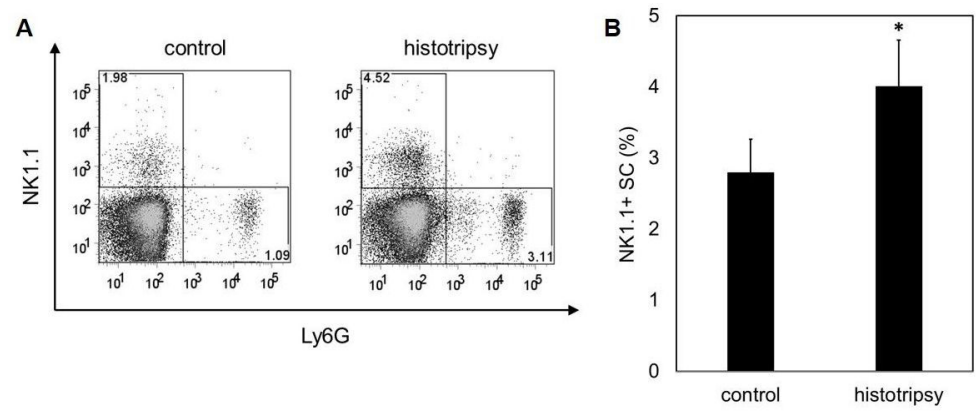

D
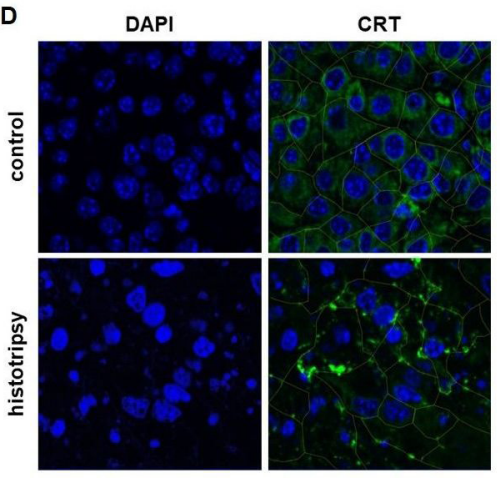

E

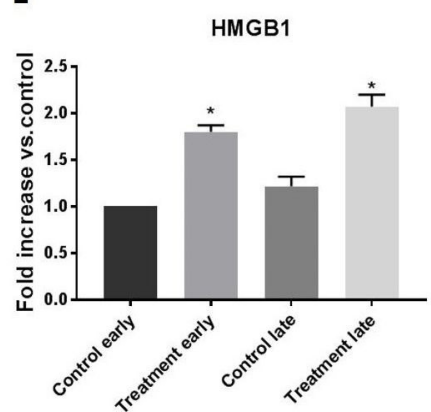

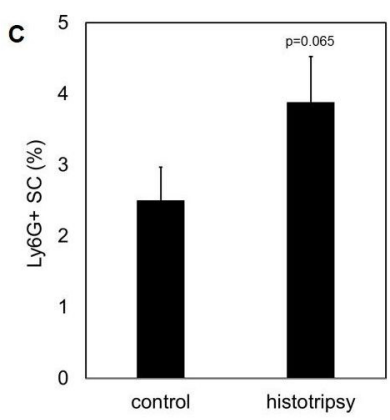

$\mathbf{F}$

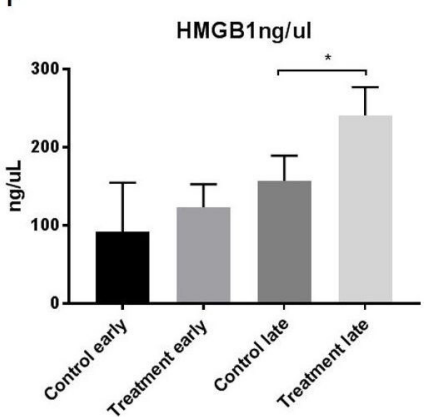

Figure 6 Histotripsy promotes local and systemic inflammatory events and tumorous release of damage-associated molecular patterns. Ten days after B16GP33 melanoma tumor inoculation, C57BL/6 mice were treated with sham or histotripsy tumor ablation. Fluorescence-activated cell sorting (FACS) analysis of splenocytes (gated on viable lymphocytes) demonstrated significantly larger populations of circulating NK1.1+ natural killer cells and a trend toward larger populations of Ly6G+ neutrophils (A-C) 10 days after histotripsy. Immunofluorescence analyses of tumors confirmed colocalization of CRT with the endoplasmic reticulum protein ERp72 (data not shown); 1 day after histotripsy ablation, DAPI staining identified marked nuclear condensation and translocation of CRT to the plasma membrane (outlined in green) consistent with apoptosis induction (D). Immunofluorescence analysis of tumors also showed significant increases in levels of extracellular HMGB1 on day 1 (early) and day 7 (late) after histotripsy (treatment) (E). By day 7 after histotripsy, serum ELISA identified significantly higher levels of circulating HMGB1 (F) ( $n=3$ per group, ${ }^{*} \mathrm{p}<0.05$ vs control by t-test). CRT, calreticulin; HMGB1, high mobility group box protein 1. 
the intratumoral microenvironment compared with untreated controls (figure 6E, online supplementary figure 2). By day 7 after histotripsy treatment, ELISA (figure 6F) measured significant increases in serum levels of HMGB1 compared with untreated controls.

\section{Histotripsy augments the efficacy of checkpoint inhibition immunotherapy}

We tested the ability of histotripsy-associated antitumor immune stimulation to work cooperatively with conventional immunotherapy. C57BL/6 mice were inoculated with bilateral subcutaneous B16GP33 melanoma tumors, then treated with sham or intraperitoneal injections of anti-CTLA- 4 mAb on days 6,9 , and 12 , and sham or histotripsy ablation on day 7 . As shown in figure $7 \mathrm{~A}$, the systemic ability of checkpoint inhibition to control tumor growth was slightly stronger than the abscopal effect of histotripsy ablation. When used together, the addition of contralateral histotripsy significantly augmented the therapeutic efficacy of checkpoint inhibition. As demonstrated in figure 7B,C, checkpoint inhibition, unilateral histotripsy, and combination therapy were all associated with significant increases in CD8+ tumor-infiltrating

B

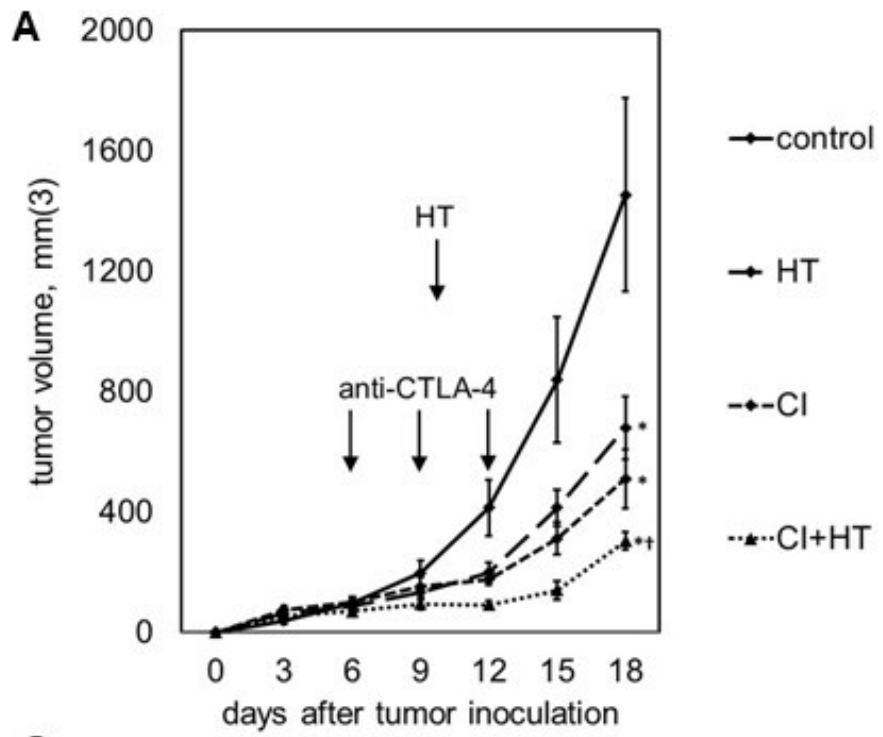

C

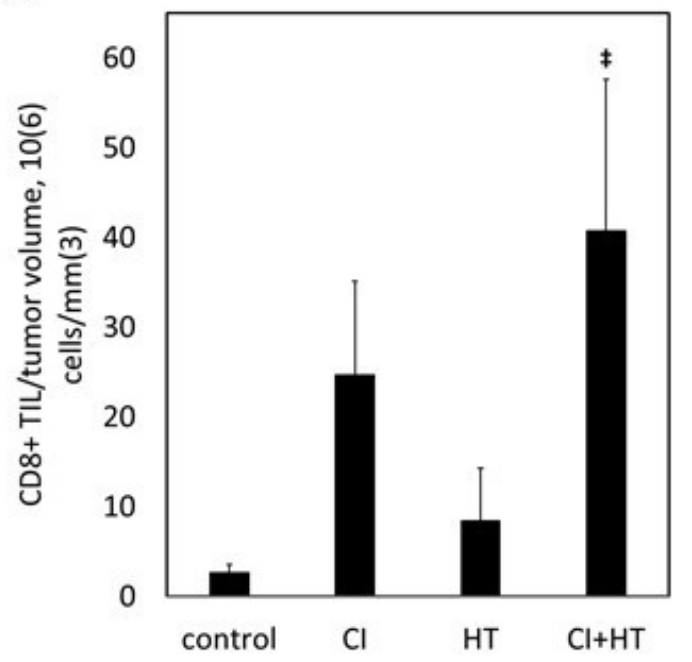

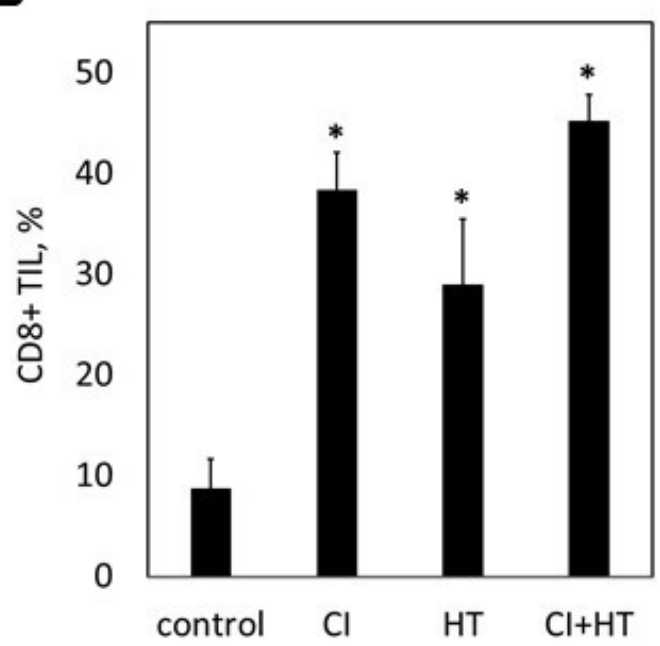

D

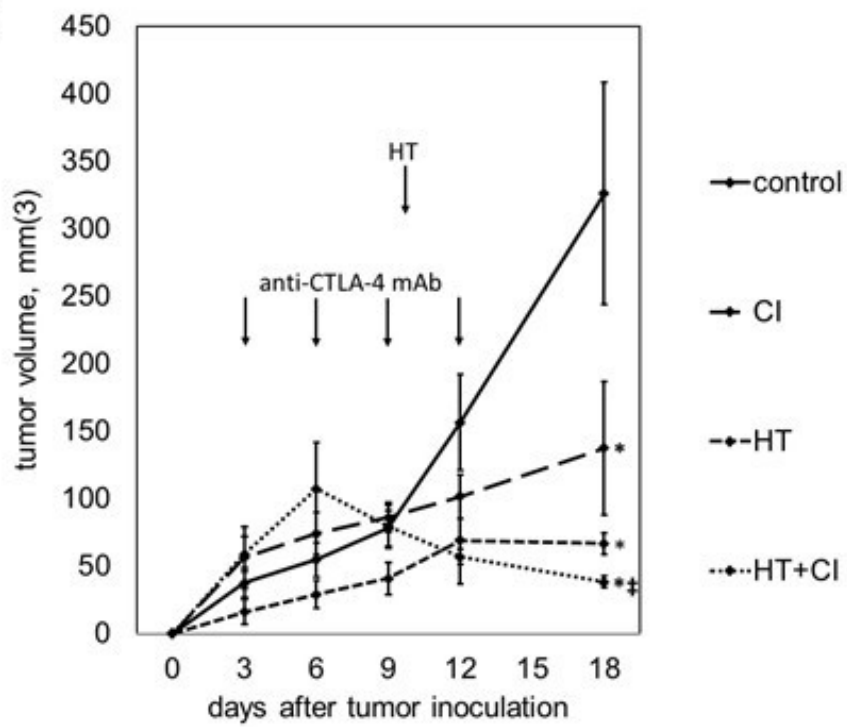

Figure 7 Histotripsy augments the therapeutic efficacy of checkpoint inhibition. C57BL/6 mice bearing bilateral B16GP33 tumors were treated with or without anti-cytotoxic T lymphocyte-associated protein-4 (CTLA-4) mAb (Cl) on days 6, 9 and 12 , and with or without unilateral histotripsy tumor ablation $(\mathrm{HT})$ on day 7 . Serial tumor measurements demonstrated optimal suppression of non-ablated tumors in mice receiving combination therapy $(A)$. When measured as a percentage of tumor infiltrating lymphocytes (TIL), intratumoral CD8+ T cells were increased in non-ablated tumors in all treatment groups (B); however, when measured as cell numbers normalized to tumor volume, significant increases in intratumoral CD8+ T cells within non-ablated tumors were only observed after combination therapy (C). C57BL/6 mice bearing bilateral Hepa1-6 tumors were treated with or without anti-CTLA-4 mAb on days 3, 6, 9 and 12, and with or without unilateral tumor ablation on day 10 . Serial tumor measurements demonstrated optimal suppression of non-ablated tumors in mice receiving combination therapy $(D)(n=4$ per group; ${ }^{*} p<0.05$ vs control by t-test; $\nmid p<0.05$ vs histotripsy by t-test; $\neq p<0.05$ vs all other groups by analysis of variance). 
lymphocytes in contralateral, non-ablated tumors; however, when quantified as numbers of infiltrating CD8+ T cells normalized to tumor volume, the addition of unilateral histotripsy significantly increased CD8+ TIL concentration compared with checkpoint inhibition alone. To verify that histotripsy was able to potentiate immune responses against less immunogenic tumors, C57BL/6 mice were inoculated with bilateral subcutaneous Hepa1-6 hepatocellular carcinoma tumors, then treated with sham or intraperitoneal injections of anti-CTLA-4 mAb on days 3, 6, 9 and 12, and sham or histotripsy ablation on day 10 . As shown in figure 7D, contralateral histotripsy stimulated abscopal antitumor immune effects that produced a similar enhancement of checkpoint inhibition immunotherapeutic efficacy in this more poorly immunogenic tumor model.

\section{DISCUSSION}

The ability of checkpoint inhibition to thwart tumorassociated immune suppression has revolutionized the care and prognosis of patients with relatively immunogenic malignancies like melanoma and lung cancer. ${ }^{23-29}$ However, overall response rates with checkpoint inhibition are limited, and the success of immunotherapy has not been fully translated to patients with less immunogenic cancers. ${ }^{30-33}$ Efforts to increase the impact of checkpoint inhibition may require immunomodulatory interventions that can magnify tumor immunogenicity. The ability of radiation therapy and thermal ablation to trigger tumordirected immune responses has been explored for years, but the magnitude of their immunostimulation and their ability to meaningfully impact cancer immunotherapy remain undetermined. ${ }^{1-8}$ Promising studies have also suggested that high-intensity focused ultrasound (HIFU), which uses ultrasonic waves to induce thermal injury, may be capable of promoting local inflammatory and immunostimulatory effects. ${ }^{34-36}$ However, like thermal ablation, HIFU relies on the induction of denaturing heat that could theoretically blunt the immunogenicity of the posttreatment tumor microenvironment.

We observed that histotripsy ablation of subcutaneous melanoma tumors resulted in a marked upregulation of intratumoral CD8+ Tcell infiltration. The magnitude of this TIL mobilization was much greater than that seen with unfractionated ablative radiation and thermal ablation in our murine model, suggesting that the unique non-thermal mechanical tissue disruption created by histotripsy ablation may offer a qualitatively different opportunity for immunostimulation. The immunostimulatory potency of histotripsy may be related to its ability to promote immunogenic cell death. Unlike thermal ablation, which exerts its effect through heat-based denaturation and necrosis, histotripsy causes cell membrane disruption while preserving the integrity of subcellular components that could have immunogenic or proinflammatory effects on their release from tumor cells. This may explain why non-thermal histotripsy mediates immunostimulatory effects not seen with thermal radiofrequency ablation. Indeed, we observed that the nonviable tumor homogenate created by histotripsy contains peptide tumor antigens with preserved immunogenicity, whereas tumor homogenates generated after freeze-heat liquefaction do not. The unique ability of histotripsy to markedly upregulate circulating numbers of CD8+ T cells specific for the minor tumor antigen GP33 suggests that histotripsy may facilitate cross-presentation of tumor antigens. ${ }^{37-39}$ Indeed, the induction of inflammatory and antigen-presenting cell populations within the ablated tumor and the therapeutically favorable skewing of tumorspecific CD8+ effector:CD4+ regulatoryT cells within the systemic circulation suggest that the local immunostimulatory effects of histotripsy may be potent enough to globally reorient the systemic immune system toward tumor antigen recognition. Radiation therapy has been proposed as a means to promote apoptotic immunogenic cell death. ${ }^{6-8}$ Our data indicate that the magnitude of immunostimulation induced by radiation therapy is small compared with histotripsy, but further comparative analyses will be needed to definitively compare histotripsy with alternative approaches to radiation and thermal ablation. It is recognized that proapoptotic conditions of cell death in which DAMPs like CRT, HMGB1, heat shock proteins, and nucleic acids are released from dying cells are highly inflammatory, inducing the migration and activation of phagocytes and antigen-presenting cells that subsequently promote tumor antigen presentation to the adaptive immune system. In contrast, largely pronecrotic conditions of cell death in which involution of dying cells is not accompanied by the release of DAMPs or exposure of tumor antigens lead to a tolerogenic, non-immunogenic cell death. ${ }^{40-42}$ Indeed, we observed evidence of both local and systemic inflammatory responses following histotripsy, manifested by increases in intratumoral NK, $\mathrm{DC}$, neutrophil, $\mathrm{B}$ and $\mathrm{T}$ cell populations, as well as elevated levels of circulating NK cells. We also observed a striking early translocation of CRT from the cytosolic endoplasmic reticulum to the plasma membrane, and translocation of HMGB1 out of the nucleus immediately after histotripsy ablation, followed by significant increases in circulating HMGB1 levels. These phenomena suggest that the ability of histotripsy to promote release of DAMPs from dying tumor cells may be involved in the stimulation of innate and adaptive immune responses against tumor. Ongoing work in our laboratory is directed at dissecting the potentially causative role that release of HMGB1 or other DAMPs may have in histotripsy immunostimulation.

Because of this ability of histotripsy to generate systemic immune responses against tumor, we used a twotumor model to demonstrate that unilateral histotripsy tumor ablation is capable of stimulating abscopal CD8+ TIL responses within contralateral, untreated tumors. Although previous studies in various animal models have demonstrated the induction of measurable abscopal immune responses following thermal ablation and radiation, ${ }^{1-8}$ we observed that the magnitude of this systemic 
effect was much stronger with histotripsy than with radiofrequency ablation and radiation therapy in our murine model. Within the tumor microenvironment, we observed qualitative differences in the patterns of intratumoral CD8+ Tcell infiltration between treated and distant tumors. Whereas TIL populations were heavily concentrated along the periphery of ablation zones in treated tumors, TILs were localized more diffusely at distant tumor sites. The ability of subtotal (unilateral) histotripsy ablation to promote tumor-directed immune responses at both treated and untreated tumor sites resulted in a retardation of distant tumor growth. Despite the presence of contralateral tumors that were left completely untreated, this retardation was sufficient to promote statistically significant prolongation of survival. The potency of histotripsy-mediated abscopal effects was further illustrated by the ability of local histotripsy ablation to concomitantly inhibit distant pulmonary metastases. Importantly, we found that the immunostimulatory impact of histotripsy could work cooperatively with checkpoint inhibition immunotherapy, imparting a significant improvement in tumor control beyond that seen with checkpoint inhibition alone. Moreover, histotripsymediated abscopal immune responses were capable of potentiating checkpoint inhibition immunotherapy against more poorly immunogenic tumors like hepatocellular carcinoma.

Our observation that histotripsy immunostimulation could work cooperatively to enhance the efficacy of checkpoint inhibition immunotherapy against melanoma and hepatocellular carcinoma has important clinical implications. Contemporary checkpoint inhibitors like anti-CTLA-4 and anti-PD-1 monoclonal antibodies have, alone or in combination, yielded remarkable responses and prolonged survival for a subset of patients whose advanced melanoma and lung cancer had until only recently been largely untreatable. Recent studies have also suggested the therapeutic efficacy of CTLA-4 blockade for malignancies like hepatocellular carcinoma. ${ }^{42-44}$ However, a sizeable majority of patients with these diseases remain unresponsive to checkpoint inhibition, and the remarkable benefits of immunotherapy have not been widely translated to patients with other, less immunogenic malignancies. It has become well established that responsiveness to checkpoint inhibition correlates with markers of tumor immunogenicity, as reflected by mutation and neoantigen frequency. ${ }^{45} 46$ Checkpoint inhibitors are unlikely to be able to add meaningful impact in a setting of poorly immunogenic tumors that do not trigger endogenous immune responses on their own.

\section{CONCLUSIONS}

Our observations suggest that histotripsy is capable of stimulating local, regional, and systemic antitumor immune responses that, when further amplified by checkpoint inhibition, may be sufficient to make a clinical impact against previously immunoresistant cancers. If so, the immunomodulatory impact of histotripsy may be key to expanding the impact and promise of cancer immunotherapy.

\section{Author affiliations}

${ }^{1}$ Surgery, University of Michigan, Ann Arbor, Michigan, USA

${ }^{2}$ Department of Hepatobiliary Surgery, Xijing Hospital, Xian, Shaanxi, China ${ }^{3}$ Biomedical Engineering, University of Michigan, Ann Arbor, Michigan, USA

${ }^{4}$ Surgery, VA Ann Arbor Healthcare System, Ann Arbor, Michigan, USA

${ }^{5}$ Surgery, Ohio State University Medical Center, Columbus, Ohio, USA

Contributors SQ, AEF, AG, MVB, ALP, AAK, HG, JD and MT performed in vivo tumor inoculations and treatments and in vitro assays. TW and $\mathrm{RH}$ performed in vivo histotripsy treatments. HH performed HMGB1 assays. SQ, TW, AEF, AG, RH, ALP, AAK, AT, ZX and CSC performed data analysis and interpretation. SQ, TW, AEF, AT, ZX and CSC wrote the manuscript. SQ, TW, AEF, AG, MVB, RH, ALP, AAK, MT, HH, AT, ZX and CSC performed manuscript review, editing and approval.

Funding This work was funded by VA Merit Review 1I01BX001619-05 (to CSC), NIH Grant R01-CA211217 (to ZX), University of Michigan Forbes Institute for Discovery (to CSC and ZX), HistoSonics-Michigan Corporate Relations Network Grant AWD006745 (to CSC), NIH Grant T32-CA009672 (to AEF), and NIH Grant T32-CA090217 (to MVB). This work was supported by NIH Grant P30-CA04659229 to the University of Michigan Rogel Cancer Center. The content is solely the responsibility of the authors and does not represent the views of the Department of Veterans Affairs or the US Government or the National Institutes of Health.

Competing interests None declared.

Patient consent for publication Not required.

Ethics approval The murine experiments were prospectively reviewed and approved by the University of Michigan and VA Ann Arbor Healthcare Animal Care and Use Committees.

Provenance and peer review Not commissioned; externally peer reviewed. Data availability statement Data are available upon reasonable request.

Open access This is an open access article distributed in accordance with the Creative Commons Attribution Non Commercial (CC BY-NC 4.0) license, which permits others to distribute, remix, adapt, build upon this work non-commercially, and license their derivative works on different terms, provided the original work is properly cited, appropriate credit is given, any changes made indicated, and the use is non-commercial. See http://creativecommons.org/licenses/by-nc/4.0/.

\section{REFERENCES}

1 Li G, Staveley-O'Carroll KF, Kimchi ET. Potential of radiofrequency ablation in combination with immunotherapy in the treatment of hepatocellular carcinoma. J Clin Trials 2016;06.

2 Sabel MS, Su G, Griffith KA, et al. Rate of freeze alters the immunologic response after cryoablation of breast cancer. Ann Surg Oncol 2010;17:1187-93.

3 Ahmad F, Gravante G, Bhardwaj N, et al. Changes in interleukin$1 \beta$ and 6 after hepatic microwave tissue ablation compared with radiofrequency, cryotherapy and surgical resections. Am J Surg 2010;200:500-6.

4 Chu KF, Dupuy DE. Thermal ablation of tumours: biological mechanisms and advances in therapy. Nat Rev Cancer 2014;14:199-208.

5 den Brok MHMGM, Sutmuller RPM, van der Voort R, et al. In situ tumor ablation creates an antigen source for the generation of antitumor immunity. Cancer Res 2004;64:4024-9.

6 Park SS, Dong H, Liu X, et al. Pd-1 restrains radiotherapy-induced Abscopal effect. Cancer Immunol Res 2015;3:610-9.

7 Ngwa W, Irabor OC, Schoenfeld JD, et al. Using immunotherapy to boost the abscopal effect. Nat Rev Cancer 2018;18:313-22.

8 Formenti SC, Rudqvist N-P, Golden E, et al. Radiotherapy induces responses of lung cancer to CTLA-4 blockade. Nat Med 2018;24:1845-51.

9 O'Brien MA, Power DG, Clover AJ, et al. Local tumour ablative therapies: opportunities for maximising immune engagement and activation. Biochim Biophys Acta 2014;2014:510-23.

10 Mehta A, Oklu R, Sheth RA. Thermal ablative therapies and immune checkpoint modulation: can locoregional approaches effect a systemic response? Gastroenterol Res Pract 2016;2016:1-11. 
11 Hall TL, Fowlkes JB, Cain CA. A real-time measure of cavitation induced tissue disruption by ultrasound imaging Backscatter reduction. IEEE Trans Ultrason Ferroelectr Freq Control 2007:54:569-75.

12 Wang T-yin, Xu Z, Winterroth F, et al. Quantitative ultrasound Backscatter for pulsed cavitational ultrasound therapy- histotripsy. IEEE Trans Ultrason Ferroelectr Freq Control 2009;56:995-1005.

13 Wang T-Y, Hall TL, Xu Z, et al. Imaging feedback of histotripsy treatments using ultrasound shear wave elastography. IEEE Trans Ultrason Ferroelectr Freq Control 2012;59:1167-81.

14 Xu Z, Ludomirsky A, Eun LY, et al. Controlled ultrasound tissue erosion. IEEE Trans Ultrason Ferroelectr Freq Control 2004;51:726-36.

15 Parsons JE, Cain CA, Abrams GD, et al. Pulsed cavitational ultrasound therapy for controlled tissue homogenization. Ultrasound Med Biol 2006;32:115-29.

16 Xu Z, Owens G, Gordon D, et al. Noninvasive creation of an atrial septal defect by histotripsy in a canine model. Circulation 2010;121:742-9.

17 Maxwell AD, Wang T-Y, Cain CA, et al. Cavitation clouds created by shock scattering from bubbles during histotripsy. J Acoust Soc Am 2011;130:1888-98.

18 Vlaisavljevich E, Maxwell A, Mancia L, et al. Visualizing the Histotripsy process: bubble Cloud-Cancer cell interactions in a Tissue-Mimicking environment. Ultrasound Med Biol 2016;42:2466-77.

19 Russ AJ, Wentworth L, Xu K, et al. Suppression of T-cell expansion by melanoma is exerted on resting cells. Ann Surg Oncol 2011;18:3848-57.

20 Russ AJ, Xu K, Wentworth L, et al. Melanoma-induced suppression of tumor antigen-specific T cell expansion is comparable to suppression of global T cell expansion. Cell Immunol 2011;271:104-9.

21 Wentworth L, Meyers JV, Alam S, et al. Memory T cells are uniquely resistant to melanoma-induced suppression. Cancer Immunol Immunother 2013;62:149-59.

22 Contreras A, Sen S, Tatar AJ, et al. Enhanced local and systemic anti-melanoma CD8+ T cell responses after memory $\mathrm{T}$ cell-based adoptive immunotherapy in mice. Cancer Immunol Immunother 2016;65:601-11.

23 Mahvi DA, Meyers JV, Tatar AJ, et al. Ctla-4 blockade plus adoptive T-cell transfer promotes optimal melanoma immunity in mice. $J$ Immunother 2015;38:54-61.

24 Contreras A, Beems MV, Tatar AJ, et al. Co-Transfer of tumor-specific effector and memory CD8+ T cells enhances the efficacy of adoptive melanoma immunotherapy in a mouse model. $J$ Immunother Cancer 2018;6.

25 Huang $\mathrm{H}$, Chen $\mathrm{H}-\mathrm{W}$, Evankovich J, et al. Histones activate the NLRP3 inflammasome in Kupffer cells during sterile inflammatory liver injury. J.i. 2013;191:2665-79.

26 Hodi FS, O'Day SJ, McDermott DF, et al. Improved survival with ipilimumab in patients with metastatic melanoma. $N$ Engl J Med 2010;363:711-23.

27 Wolchok JD, Kluger H, Callahan MK, et al. Nivolumab plus ipilimumab in advanced melanoma. $N$ Engl $\mathrm{J}$ Med 2013;369:122-33.
28 Larkin J, Chiarion-Sileni V, Gonzalez R, et al. Combined nivolumab and ipilimumab or monotherapy in untreated melanoma. $N$ Engl $J$ Med 2015;373:23-34.

29 Brahmer J, Reckamp KL, Baas P, et al. Nivolumab versus docetaxel in advanced squamous-cell non-small-cell lung cancer. $N$ Engl $\mathrm{J}$ Med 2015;373:123-35.

30 Borghaei H, Paz-Ares L, Horn L, et al. Nivolumab versus docetaxel in advanced Nonsquamous Non-Small-Cell lung cancer. N Engl J Med 2015;373:1627-39.

31 Motzer RJ, Escudier B, McDermott DF, et al. Nivolumab versus everolimus in advanced renal-cell carcinoma. $N$ Engl J Med 2015;373:1803-13

32 Royal RE, Levy C, Turner K, et al. Phase 2 trial of single agent ipilimumab (anti-CTLA-4) for locally advanced or metastatic pancreatic adenocarcinoma. J Immunother 2010;33:828-33.

33 Lee B, Hutchinson R, Wong H-L, et al. Emerging biomarkers for immunomodulatory cancer treatment of upper gastrointestinal, pancreatic and hepatic cancers. Semin Cancer Biol 2018;52:241-52.

34 Khokhlova VA, Fowlkes JB, Roberts WW, et al. Histotripsy methods in mechanical disintegration of tissue: towards clinical applications. Int J Hyperthermia 2015;31:145-62.

35 Schade GR, Wang Y-N, D'Andrea S, et al. Boiling histotripsy ablation of renal cell carcinoma in the Eker rat promotes a systemic inflammatory response. Ultrasound Med Biol 2019;45:137-47.

36 Khokhlova T. T-Cell mediated immune response to HIFU-induced liquefaction of murine B16 melanoma. J Ther Ultrasound 2015;3.

37 Marzo AL, Lake RA, Lo D, et al. Tumor antigens are constitutively presented in the draining lymph nodes. J Immunol 1999:162:5838-45

38 Preynat-Seauve O, Contassot E, Schuler P, et al. Extralymphatic tumors prepare draining lymph nodes to invasion via a T-cell crosstolerance process. Cancer Res 2007;67:5009-16.

39 Contassot E, Preynat-Seauve O, French L, et al. Lymph node tumor metastases: more susceptible than primary tumors to CD8+ T-cell immune destruction. Trends Immunol 2009;30:569-73.

40 Krysko DV, Garg AD, Kaczmarek A, et al. Immunogenic cell death and DAMPs in cancer therapy. Nat Rev Cancer 2012;12:860-75.

41 Ladoire S, Hannani D, Vetizou M, et al. Cell-Death-Associated molecular patterns as determinants of cancer immunogenicity. Antioxid Redox Signal 2014;20:1098-116.

42 Bezu L, Gomes-de-Silva LC, Dewitte H, et al. Combinatorial strategies for the induction of immunogenic cell death. Front Immunol 2015;6.

43 Sangro B, Gomez-Martin C, de la Mata M, et al. A clinical trial of CTLA-4 blockade with tremelimumab in patients with hepatocellular carcinoma and chronic hepatitis C. J Hepatol 2013;59:81-8.

44 Agdashian D, ElGindi M, Xie C, et al. The effect of anti-CTLA4 treatment on peripheral and intra-tumoral $\mathrm{T}$ cells in patients with hepatocellular carcinoma. Cancer Immunol Immunother 2019;68:599-608.

45 Snyder A, Makarov V, Merghoub T, et al. Genetic basis for clinical response to CTLA-4 blockade in melanoma. $N$ Engl $\mathrm{J}$ Med 2014:371:2189-99.

46 Champiat S, Ferté C, Lebel-Binay S, et al. Exomics and immunogenics: bridging mutational load and immune checkpoints efficacy. Oncoimmunology 2014;3:e27817. 